\title{
Late referrals of pediatric patients with elevated blood pressure
}

\author{
Guido Filler $^{1,2}$ (D) Laura Torres-Canchala ${ }^{2,3}$
}

Received: 29 January 2020 / Accepted: 30 January 2020 / Published online: 11 February 2020

(C) IPNA 2020

\section{Untreated hypertension}

Untreated hypertension in children and adolescents can lead to disastrous consequences in the short and long term [1]. Acute rise of blood pressure by more than $30 \mathrm{mmHg}$ in children may lead to brain involvement (e.g., cognitive abnormalities, hypertensive encephalopathy), left ventricular insufficiency, visual disturbances due to damage of the retina, and epistaxis [2]. While a hypertensive crisis is rarely overlooked, milder hypertension is asymptomatic and may not be recognized, remaining untreated for years. This is despite the fact that patients may develop irreversible end-organ damage, major cardiovascular morbidity, and mortality later in life [3]. There is evidence of underdiagnosis of hypertension in children and adolescents [4]. This is especially true in the emergency department, as elevated blood pressures are noted but remain unreferred, even for adults [5]. Barriers to referral for elevated blood pressure include education about reference intervals, hesitancy if patients are asymptomatic, required financial compensation to refer, and a perception that blood pressure was influenced by the medical doctor [5]. While the referral hesitancy applies equally to nurses, physician assistants, and emergency physicians, the latter group also cites lack of time as a barrier, doubts about their concern for the blood pressure, and feeling emotionally uncomfortable when referring [5]. Children and adolescents with elevated blood pressure are often referred to subspecialists, namely pediatric

\section{Guido Filler}

guido.filler@lhsc.on.ca

1 Departments of Paediatrics, Medicine and Pathology and Laboratory Medicine, Paediatric Nephrology, Schulich School of Medicine \& Dentistry, Children's Hospital, London Health Sciences Centre, University of Western Ontario, 800 Commissioners Road East, London, Ontario N6A 5W9, Canada

2 Lilibeth Caberto Kidney Clinical Research Unit, London, Ontario, Canada

3 Centro de Investigaciones Clínicas, Fundación Valle del Lili, Cali, Colombia nephrologists or pediatric cardiologists, owing to the need for a detailed work-up for underlying causes [6], whereas adults most often have essential hypertension; secondary etiologies are more common in the pediatric setting.

Owing to the obesity pandemic, hypertension is on the rise among children [7] around the globe. Obesity is a major predictor of childhood hypertension, and in younger children more so than other known risk factors such as low birth weight [8]. Unfortunately, there is a paucity of data in the literature about recognition of hypertension in children and time to referral to specialists or subspecialists who treat hypertension. This is in spite of published access targets for the diagnosis and management of hypertension $[9,10]$. In this context, we are pleased to read in this edition of Pediatric Nephrology Hamby's study of 120 children and adolescents from a single pediatric nephrology practice in Fort Worth, North Texas. Their cohort was analyzed in depth about the time to referral to the pediatric nephrology team from the first documented elevated blood pressure [11].

\section{The study by Tyler Hamby et al.}

For their study, Hamby et al. used a convenient sample of patients who had one or more office blood pressure records prior to their hypertension clinic referral in a single-center retrospective study. Patients were aged 3-18 years and were collected from July 2014 to June 2017. Data were obtained from the electronic medical record (EMR) system, which are now used routinely among developed, emerging, and even some developing countries. The authors used the current guidelines to define elevated blood pressure and hypertension [12]. All patients had at least one elevated blood pressure and the majority of patients had more than 3 elevated blood pressures recorded. Most patients reached criteria for hypertension prior to referral, but $60 \%$ were referred more than 1 year later, and in one case, the time to referral was $>10$ years. The median number of visits prior to referral was 5 after one episode of elevated blood pressure and 4 visits after 3 episodes of 
elevated blood pressure. Most of the referrals came from primary care, followed by specialty care, and only $14 \%$ of referrals came from the emergency room. The study offers some more detailed insight into the patient cohort and reports on the socio-economic status of the patients and ethnicity, but of all parameters, only obesity was significant. However, this only applied if patients had at least three elevated blood pressures on record.

Hamby and team noted that obesity was a major predictor among those patients who had at least 3 documented elevated blood pressure readings. It is well documented that obesity is a major risk factor for hypertension $[8,13,14]$. The guidelines clearly recommend checking blood pressure if the patients have a family history of essential hypertension or if they are overweight or obese [12].

\section{Strengths and limitations of Hamby's study}

The paper may represent a snapshot of the reality, namely that elevated blood pressure in primary care, other clinics, or the emergency room are either not reacted upon appropriately or possibly not even recognized. The study is in keeping with previous literature about underrecognized hypertension in the pediatric age group [4]. Moreover, Hamby's study quantitates the time from $>3$ elevated blood pressure readings to referral at a median of 4 years, which is a frightening statistic. The study limitations include a retrospective sample of convenience, preventing generalization. The authors did not account for patients being treated elsewhere and acknowledge that patients may have been sent to pediatric cardiologists instead. They did not list the indications for referral for a pediatric nephrologist. It would have been nice to know how many of the patients had already developed end-organ damage, such as left ventricular hypertrophy or retinal changes secondary to the undiagnosed/untreated hypertension. There is also no information as to how elevated blood pressure is flagged in the EMR.

\section{What are the reasons for the late referral?}

The study points to the problem of recognizing hypertension and/or hesitancy of referral. Most EMRs nowadays have the ability to flag elevated blood pressures. It is acknowledged that the current blood pressure tables are complicated. Without the support of an EMR that automatically calculates blood pressure $z$-scores from age, height, and gender, the busy primary care physician or emergency room physician may not be able to quickly classify a blood pressure reading as abnormal. Simplified blood pressure tables may serve as simpler tool to accurately classify office blood pressure [15]. Furthermore, the use of smart phone apps such as $\operatorname{Ped}(\mathrm{z})$ (https://www.pedz.de/en/welcome.html) may help identify elevated blood pressure readings, but this requires another step for the already overloaded clinical practitioners. However, this does not address the hesitancy.

It is acknowledged that blood pressure screening of all children may not be feasible. Targeted screening may be more feasible, based on overweight may be a good strategy. A recent diagnostic model that includes maternal hypertension during pregnancy, maternal BMI, maternal education level, parental hypertension, parental smoking, childbirth standard deviation score, child BMI $z$-score, and child ethnicity improved targeted blood pressure monitoring of children at risk [16].

It is standard of care for any pediatric clinic visit to obtain height and weight of the patients. The EMR, if used, should produce information about the body mass index percentiles. Also, obesity is easy to diagnose clinically, especially if there are striae, acanthosis nigricans, breathlessness, increased sweating, snoring, inability to cope with sudden physical activity, etc. Hesitancy of referral should therefore not be a factor in obese children. In a recent meta-analysis, the prevalence of hypertension for obese children was $15.27 \%, 4.99 \%$ for overweight children, and only $1.90 \%$ in normal weight children [7]. In other words, one of six obese children or adolescents has hypertension. It has been shown that masked hypertension is more prevalent among obese children as well, and thus, whenever possible, obese children should undergo 24-h ambulatory blood pressure monitoring [17]. Unfortunately, availability of 24-h ambulatory blood pressure monitoring is variable. Moreover, 24-h ambulatory blood pressure is not reimbursed in most Canadian provinces and in many other parts of the world. It has been pointed out that the diagnosis of masked hypertension may help to reduce cardiovascular morbidity [17].

Overall, the reasons for late referral of hypertensive children and adolescents remain understudied. Future work has to focus on the various aspects of elevated blood pressure identification, such as difficulties with interpreting blood pressure readings, education, EMR-related problems (not intrinsic calculation of blood pressure percentiles based on sex and height percentiles), and provider-related factors that may lead to hesitancy. We are only aware of Souffront's study which assessed in detail the reasons for lack of referrals in the adult emergency room [5], on a survey of 450 adult emergency room physicians. Pediatric data should be obtained. The reasons for the failure of other outpatient clinics to refer for hypertension remain unknown. In our own center, blood pressure is often not even measured outside of the nephrology and cardiology clinics. However, it is regularly measured in the emergency room.

\section{Conclusion}

Taken together, referrals for milder cases of hypertension, especially obesity-related, are occurring late. Hamby's study 
contributes to the literature by providing preliminary data about the time to referral to the hypertension clinics. Given the potentially significant benefit of early treatment of hypertension in children to reduce long-term morbidity and mortality, the reasons of underdiagnosing hypertension and late referral for diagnosis and treatment need to be determined. This will identify potential targets of intervention to remove barriers for adequate referral. Hamby and colleagues should be congratulated for addressing this vastly understudied problem.

Acknowledgments We thank Dr. Maria Ferris for her thorough review of the manuscript.

\section{References}

1. Hope KD, Zachariah JP (2019) Predictors and consequences of pediatric hypertension: have advanced echocardiography and vascular testing arrived? Curr Hypertens Rep 21:54

2. Muller-Wiefel DE (1988) The hypertensive crisis in childhood. Wien Klin Wochenschr 100:547-555

3. Middeke MR (1997) Office hypertension-early stage of hypertension. Epidemiology, causes and consequences. Fortschr Med 115: 26-28 30, 32 passim

4. Hansen ML, Gunn PW, Kaelber DC (2007) Underdiagnosis of hypertension in children and adolescents. JAMA 298:874-879

5. Souffront K, Chyun D, Kovner C (2015) Barriers to referral for elevated blood pressure in the emergency department and differences between provider type. J Clin Hypertens (Greenwich) 17: 207-214

6. Flynn JT, Kaelber DC, Baker-Smith CM et al (2017) Subcommittee on Screening and Management of High Blood Pressure in Children. Clinical practice guideline for screening and management of high blood pressure in children and adolescents. Pediatrics 140(3): e20171904. (2018) Pediatrics 142:e20181739. https://doi.org/10. 1542/peds.2018-1739

7. Song P, Zhang Y, Yu J, Zha M, Zhu Y, Rahimi K, Rudan I (2019) Global prevalence of hypertension in children: a systematic review and meta-analysis. JAMA Pediatr. https://doi.org/10.1001/ jamapediatrics.2019.3310
8. Filler G, Yasin A, Kesarwani P, Garg AX, Lindsay R, Sharma AP (2011) Big mother or small baby: which predicts hypertension? J Clin Hypertens (Greenwich) 13:35-41

9. Radina M, Sharma AP, Yasin A, Filler G (2010) Adherence to waiting-time targets for pediatric nephrology clinic referrals. Pediatr Nephrol 25:311-316

10. Filler G, Chavannes M, Yasin A (2013) The need for ongoing monitoring of adherence to access targets. Pediatr Nephrol 28: 831-832

11. Hamby T, Pueringer MR, Noorani S, Khanna A, Barrow J, Razzouk $\mathrm{R}$ (2020) Time to referral to a nephrology clinic for pediatric hypertension. Pediatr Nephrol. https://doi.org/10.1007/s00467-02004490-x

12. Flynn JT, Kaelber DC, Baker-Smith CM, Blowey D, Carroll AE, Daniels SR, de Ferranti SD, Dionne JM, Falkner B, Flinn SK, Gidding SS, Goodwin C, Leu MG, Powers ME, Rea C, Samuels J, Simasek M, Thaker VV, Urbina EM, SUBCOMMITTEE ON SCREENING AND MANAGEMENT OF HIGH BLOOD PRESSURE IN CHILDREN (2017) Clinical practice guideline for screening and management of high blood pressure in children and adolescents. Pediatrics 140:e20171904. https://doi.org/10. 1542/peds.2017-1904

13. Brady TM (2017) Obesity-related hypertension in children. Front Pediatr 5:197

14. Falkner B, Gidding SS, Portman R, Rosner B (2008) Blood pressure variability and classification of prehypertension and hypertension in adolescence. Pediatrics 122:238-242

15. Sharma AP, Mohammed J, Thomas B, Singh RN, Filler G (2015) Using simplified blood pressure tables to avoid underdiagnosing childhood hypertension. Paediatr Child Health 20:297-301

16. Hamoen M, Welten M, Nieboer D, Heymans MW, Twisk JW, Raat H, Vergouwe Y, Wijga AH, de Kroon ML (2020) Development of a prediction model to target screening for high blood pressure in children. Prev Med 132:105997

17. Huang Z, Sharman JE, Fonseca R, Park C, Chaturvedi N, Davey Smith G, Howe LD, Lawlor DA, Hughes AD, Schultz MG (2020) Masked hypertension and submaximal exercise blood pressure among adolescents from the Avon Longitudinal Study of Parents and Children (ALSPAC). Scand J Med Sci Sports 30:25-30

Publisher's note Springer Nature remains neutral with regard to jurisdictional claims in published maps and institutional affiliations. 